\title{
Purificación de una proteína de 35 kDa rica en lisina, de la fracción albúmina de Amaranthus caudatus (kiwicha)
}

\author{
Orlinda Villanueva ${ }^{1}$, Inés Arnao ${ }^{2}$
}

Resumen

Palabras clave

\begin{abstract}
Objetivo: Purificar una proteína rica en lisina de la fracción albúmina del grano de Amaranthus caudatus. Diseño: Estudio observacional descriptivo. Lugar: Centro de Investigación de Bioquímica y Nutrición, Facultad de Medicina, Universidad Nacional Mayor de San Marcos. Material biológico: Semillas de amaranto (Amaranthus caudatus), variedad Oscar Blanco. Intervenciones: Se empleó técnicas cromatográficas (filtración en gel) y electroforéticas (SDS-PAGE y electroelución) para purificar dicha proteína y determinar su peso molecular. Asimismo, se analizó su composición de aminoácidos por cromatografía líquida de alta performance (HPLC). Principales medidas de resultados: Aislamiento y purificación de una proteína de 35 kDa, rica en lisina. Resultados: Se aisló una proteína con peso molecular de $35 \mathrm{kDa}$, según PAGE-SDS, y la composición en aminoácidos esenciales fue similar a la proteína estándar recomendada por la Organización Mundial de la Salud-OMS, con un alto contenido de lisina (8,61 moles \%). Conclusiones: Se ha aislado y purificado una proteina de $35 \mathrm{kDa}$, rica en lisina de la fracción albúmina de Amaranthus caudatus, con una composición en aminoácidos esenciales comparable a lo recomendado por la OMS.
\end{abstract}

Amaranthus; albúminas; lisina; aminoácidos; aminoácidos esenciales.
Purification of a $35 \mathrm{kDa}$ protein rich in lysine from Amaranthus caudatus (kiwicha) albumin fraction Abstract

Objective: To purify a protein rich in lysine from Amaranthus caudatus seeds' albumin fraction. Design: Observational descriptive study. Setting: Biochemistry and Nutrition Research Center, Faculty of Medicine, Universidad Nacional Mayor de San Marcos. Biologic material: Amaranto (Amaranthus caudatus) seeds, Oscar Blanco variety. Interventions: We used both chromatographic (gel filtration) and electrophoretic (SDS$P A G E$ and electroelution) techniques to purify a protein rich in lysine and to determine its molecular weight. The aminoacid composition of the lysine rich protein was obtained by high pressure liquid chromatography (HPLC). Main outcome measures: Isolation and purification of a $35 \mathrm{kDa}$ protein rich in lysine. Results: We isolated a 35 $k D a$ molecular weight protein as determined by SDS$P A G E$; the essential amino acid composition was similar to the standard protein recommended by the World Health

1 Departamento de Microbiología y Biotecnología. Facultad de Ciencias, Universidad Nacional del Santa. Chimbote, Perú.

2 Centro de Investigación de Bioquímica y Nutrición. Facultad de Medicina, Universidad Nacional Mayor de San Marcos. Lima, Perú.
Organization (WHO) with high content of lysine $(8,61$ moles \%). Conclusions: We have isolated and purified a $35 \mathrm{kDa}$ protein rich in lysine from Amaranthus caudatus albumin fraction with an essential amino acid composition similar to that recommended by WHO.

Key words: Amaranthus; albumins; lysine; amino acids; amino acids, essential.

\section{INTRODUCCIÓN}

Los cultivos andinos son básicos en la canasta alimentaria de la población andina $\left(^{1}\right)$ e incluyen cereales, leguminosas, tubérculos, raíces y frutos. Sin embargo, actualmente la producción y el consumo de muchas de estas especies disminuyen considerablemente, debido a la influencia de los hábitos alimenticios de países desarrollados y a la falta de conocimientos de la calidad nutritiva de estos cultivos, que son considerados como 'alimento para la gente pobre'. Por otro lado, no hay una relación directa entre el crecimiento poblacional y el agrícola. Estas razones hacen 
necesaria la búsqueda de nuevas fuentes de proteínas o mejorar la calidad de las que ya existen, para satisfacer las necesidades de una dieta con una composición de aminoácidos balanceada para el ser humano.

El amaranto (kiwicha) es un pseudocereal que ha sido identificado como un cultivo alimentario muy prometedor, debido a su excepcional valor nutritivo por su contenido de proteínas, lípidos y minerales. Una de las características más importantes del grano es que su contenido de proteínas de almacenamiento es más alto y mejor balanceado en aminoácidos esenciales que el de los cereales $(2,3)$.

En el Perú, la especie que más se cultiva es el Amaranthus caudatus, conocido como 'kiwicha', la cual es una planta de aprovechamiento integral; sus hojas se consumen como verdura, mientras que las semillas son usadas como cereales. Su contenido de proteína cruda oscila entre 13 y $17 \%\left({ }^{4}\right)$, es relativamente rica en lisina, triptófano y metionina y pobre en leucina e isoleucina $\left.{ }^{(5-7}\right)$. Esto hace que el grano de amaranto sea un excelente complemento para los cereales (arroz, maíz, trigo), los cuales son deficientes en lisina $\left({ }^{8-10}\right)$.

Las proteínas de almacenamiento más importantes en amaranto son albúminas y globulinas, que representan entre 60 y $70 \%$ del nitrógeno proteínico total en el grano, y las glutelinas, que se hallan en menor porcentaje $\left({ }^{11}\right)$. Existe mucho interés por el estudio de estas proteínas, no solo por su reconocido valor nutricional, sino también por ciertas características que permiten su aplicación en otros campos. Tal es el caso de la globulina, que es un excelente agente emulsificante, estable al calor $\left({ }^{11}\right)$.

Se ha hecho estudios de composición de aminoácidos y patrones electroforéticos de las fracciones proteicas totales presentes en el grano (albúminas, globulinas, prolaminas, glutelinas) $\left({ }^{12,13}\right)$, así como de perfil de carga, asociación covalente de subunidades $\left({ }^{14}\right)$ y estudios calorimétricos y electroforéticos $\left({ }^{15}\right)$.

La literatura revisada señala que la fracción albúmina es la que tiene el contenido más alto en lisina $\left({ }^{5,12}\right)$. Sin embargo, son muy escasos los estudios bioquímicos referentes a la calidad y cantidad de proteínas que contribuyen a este elevado nivel. Por tal motivo, realizamos la presente investigación, con el objeto de aislar y purificar una de las proteínas más abundante de la fracción albúmina de Amaranthus caudatus, haciendo uso de técnicas cromatográficas y electroforéticas, y determinar su composición en aminoácidos por cromatografía líquida de alto perfomance (HPLC).

\section{MÉTODOS}

Se realizó un estudio observacional descriptivo, empleándose como material biológico semillas de amaranto (Amaranthus caudatus), variedad Oscar Blanco, procedente de Huaraz y proporcionada por el Programa de Cereales de la Universidad Nacional Agraria La Molina.

Las semillas fueron molidas y la harina resultante deslipidada con éter de petróleo, tamizada a través de una malla número 60 y secada a temperatura ambiente. Se almacenó a $4{ }^{\circ} \mathrm{C}$ hasta antes de su uso.

Para la extracción de la fracción albúmina, se utilizó el método de Osborne $\left({ }^{16}\right)$, con algunas modificaciones.

La harina deslipidada, en una proporción 1:3 con agua desionizada y $0,1 \mathrm{mM}$ de fenilmetilsulfonilfluoruro (PMSF), fue agitada por tres horas, a $4{ }^{\circ} \mathrm{C}$, para así extraer la fracción albúmina. La mezcla fue centrifugada a $3000 \mathrm{~g}$, por 10 minutos, y el sobrenadante liofilizado y conservado a $4{ }^{\circ} \mathrm{C}$.

Unos $60 \mathrm{mg}$ del extracto de albúmina liofilizado disueltos en el buffer de corrida (Tris $\mathrm{HCl}$ $0,1 \mathrm{M} \mathrm{pH} \mathrm{8,5)} \mathrm{fueron} \mathrm{aplicados} \mathrm{a} \mathrm{una} \mathrm{columna}$ de Sephadex G-75, con un flujo de $11 \mathrm{~mL} / \mathrm{h}$ y a temperatura de $4^{\circ} \mathrm{C}$.

Los eluatos con mayor absorbancia, a $280 \mathrm{~nm}$ del pico 1 , fueron reunidos y liofilizados.

El siguiente paso consistió en una electroelución, según lo descrito por Harrington $\left({ }^{17}\right)$, para lo cual se tomó $250 \mathrm{mg}$ del liofilizado del pico 1 y se los sometió a electroforesis preparativa en gel de 
poliacrilamida, en presencia de dodecil sulfato de sodio (PAGE-SDS), con gradiente de 5 a $20 \%$ de acrilamida. El gel fue revelado con una solución de cloruro de potasio $0,25 \mathrm{M}$ y ditiotreitol $1 \mathrm{mM}$, a $4{ }^{\circ} \mathrm{C}$. La banda proteica de interés fue cortada en pequeños trocitos y electroeluida a través de una membrana de millipore. La corrida fue por 90 minutos, con una intensidad de $30 \mathrm{~mA}$, usando un buffer de elusión con bicarbonato de amonio $50 \mathrm{mM}$ y SDS $0,1 \%(\mathrm{p} / \mathrm{v})$. La proteína de $35 \mathrm{kDa}$ electroeluida fue dializada y liofilizada.

El seguimiento del proceso de purificación y pureza de la proteína se realizó mediante electroforesis unidimensional en geles de poliacrilamida, en presencia de SDS, según el método descrito por Laemmli $\left({ }^{18}\right)$, utilizándose como marcadores albúmina de suero bovino (BSA), ovoalbúmina, mioglobina y citocromo $\mathrm{C}$.

A fin de determinar la composición en aminoácidos de la proteína purificada mediante electroelución, ésta fue hidrolizada con $\mathrm{HCl} 6 \mathrm{~N}$ y fenol $1 \%$, por 24 horas, a $110^{\circ} \mathrm{C}$ y en vacío. Luego, fue derivatizada con fenil iso tiocianato (PITC), según lo descrito por Bidlingmeyer y col. $\left({ }^{19}\right)$.

Los aminoácidos-PITC fueron analizados por HPLC, según lo descrito por Espinoza E $\left({ }^{20}\right)$.

\section{RESULTADOS}

Mediante los procedimientos antes descritos, se logró aislar y purificar una proteína de la fracción albúmina de $A$. caudatus. El patrón cromatográfico de la fracción albúmina se muestra en la Figura 1, en donde se observa 2 picos: I y II. El pico I salió con el volumen de exclusión (Vo) de la columna y los tubos de mayor absorbancia a $280 \mathrm{~nm}$ fueron juntados, liofilizados y conservados a $-20^{\circ} \mathrm{C}$ hasta antes de su uso.

La Figura 2 nos muestra los resultados de las diferentes etapas de la purificación de la proteína de $A$. caudatus. El canal D del electroforetograma nos revela la presencia de un grupo de proteínas heterogéneas con pesos moleculares entre 80 y 10 $\mathrm{kDa}$, de la fracción albúmina; estas últimas de peso molecular bajo no bien resueltas.

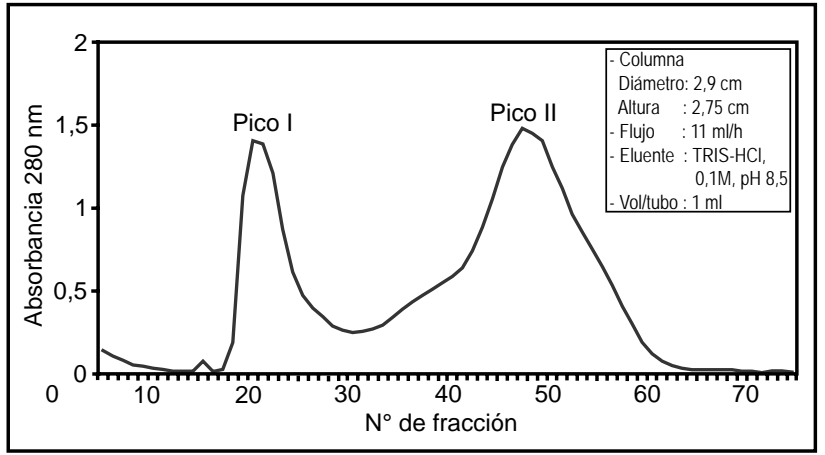

Figura 1. Cromatografía por filtración en gel de la fracción albúmina de Amaranthus caudatus.

El pico I resultante de la cromatografía en gel filtración presenta 22 bandas proteicas (banda C), siendo una de las más abundantes la de 35 $\mathrm{kDa}$. Ésta proteína electroeluida se muestra en 2 concentraciones diferentes en los canales B y B', como una banda única de peso molecular $35+/-1$ $\mathrm{kDa}$, que corresponde a la proteína purificada de la fracción albúmina de $A$. caudatus.

La composición en aminoácidos de la harina deslipidada, de la fracción albúmina y de la proteína de $35 \mathrm{kDa}$ de $A$. caudatus, puede ser observada en la Tabla 1. Hay un aumento en el contenido

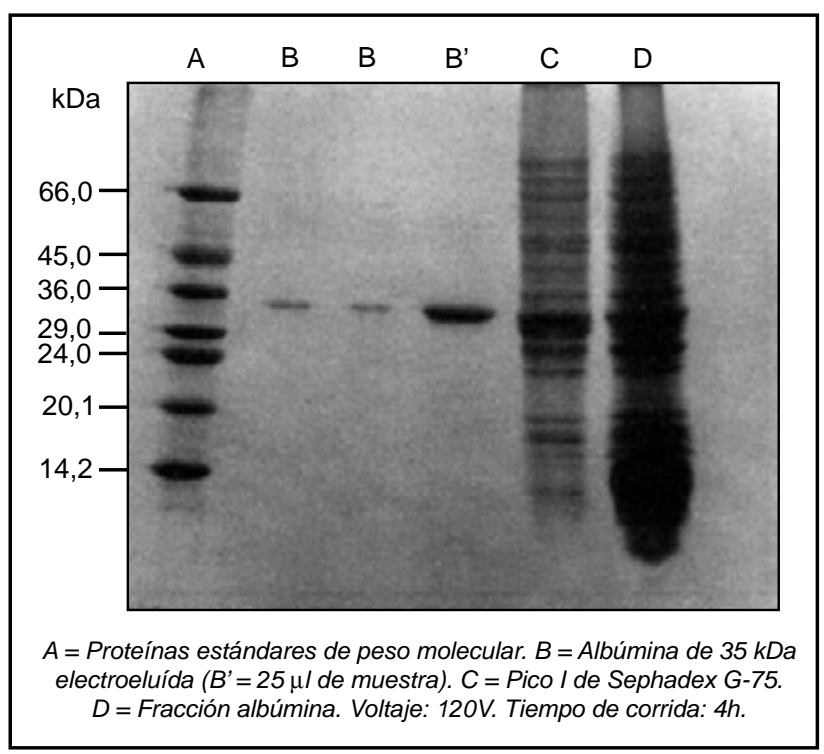

Figura 2. Electroforesis en gel de poliacrilamida en gradiente 5 - 20\% (SDS-PAGE). 
Tabla 1. Niveles de los aminoácidos de Amaranthus caudatus determinados mediante HPLC.

\begin{tabular}{lccc}
\hline Aminoácido & $\begin{array}{c}\text { Harina } \\
\text { deslipidada } \\
(\text { moles \% })\end{array}$ & $\begin{array}{c}\text { Fracción } \\
\text { albúmina } \\
(\text { moles \%) }\end{array}$ & $\begin{array}{c}\text { Proteína aislada } \\
\text { de 35 kDa } \\
(\text { moles \%) }\end{array}$ \\
\hline ASP & 2,22 & 1,85 & 12,12 \\
GLU & 18,08 & 13,38 & 10,07 \\
SER & 6,13 & 7,24 & 6,69 \\
GLI & 21,94 & 17,43 & 8,99 \\
HIS & 3,69 & 4,09 & 2,15 \\
TRE/ALA & 5,26 & 7,22 & 10,86 \\
ARG & 5,26 & 8,93 & 2,90 \\
PRO & 9,67 & 9,65 & 5,44 \\
TIR & 4,14 & 3,25 & 3,92 \\
VAL & 4,34 & 6,33 & 6,38 \\
MET & 5,11 & 4,56 & 1,83 \\
ILE & 4,68 & 5,21 & 4,92 \\
LEU & 4,81 & 5,92 & 9,55 \\
FEN & 3,12 & 2,27 & 5,60 \\
LIS & 1,55 & 2,66 & 8,61 \\
\hline
\end{tabular}

de los aminoácidos esenciales -lisina, fenilalanina y leucina- y una disminución en el aminoácido azufrado metionina, en la proteína purificada, con respecto a la harina deslipidada y a la fracción albúmina.

En la Tabla 2 se registra la composición aminoacídica de las proteínas de $35 \mathrm{kDa}$ purificadas de Amaranthus, en comparación con la proteína patrón de FAO/OMS ${ }^{(21)}$, siendo estos valores comparables. No se determinó el triptófano, porque se destruye con la hidrólisis ácida empleada en este caso.

\section{DISCUSIÓN}

Existen diversos estudios sobre la composición química del grano de amaranto y todos ellos muestran una gran variación, que depende de la variabilidad genética del material, edad de maduración de la planta, localización del cultivo y fertilidad del suelo $\left({ }^{22,23}\right)$. Nosotros hemos determinado que la concentración de proteínas de la harina deslipidada de Amaranthus caudatus es $12,8 \%$ y está dentro del rango considerado para este género, que es de 12 a $17,9 \%\left({ }^{4,24}\right)$, siendo superior al de los cereales, como el trigo.
En base a sus propiedades de solubilidad, Osborne $\left({ }^{16}\right)$ clasificó a las proteínas de las plantas en cuatro grupos: 1) albúminas, solubles en agua; 2) globulinas, solubles en soluciones salinas diluidas; 3) prolaminas, solubles en alcohol; y, 4) glutelinas, solubles en ácidos o álcalis diluidos. En el amaranto, las albúminas y globulinas representan entre $60 \%$ y $70 \%$ del nitrógeno proteínico total, siendo para algunos investigadores la albúmina la fracción de reserva proteica más abundante $\left({ }^{5,12,25}\right)$ $\mathrm{y}$, para otros, la globulina $\left({ }^{4}\right)$. Esto lo diferencia de la mayoría de cereales, en donde las principales proteínas de almacenamiento son las prolaminas; pero, es parecido al arroz y avena, donde el contenido de prolaminas es bajo (2 a 10\%) $\left.{ }^{(26}\right)$.

La calidad de las proteínas de las semillas es determinada primariamente por las proteínas de almacenamiento. Por tal motivo, nos propusimos aislar una de las principales proteínas de la fracción albúmina de Amaranthus caudatus, teniendo conocimiento que dicha fracción tiene un alto nivel de lisina, aminoácido esencial deficiente en la mayoría de cereales.

La separación de los diferentes tipos de proteína que componen la fracción albúmina, mediante cromatografía por filtración en gel, muestra un perfil de elución con dos picos, muy similar a lo descri-

Tabla 2. Comparación de los contenidos de aminoácidos esenciales de la proteína aislada de Amaranthus caudatus con otras proteínas.

\begin{tabular}{lcccc}
\hline $\begin{array}{c}\text { Aminoácidos } \\
\text { esenciales } \\
(\text { moles \%) }\end{array}$ & $\begin{array}{c}\text { A.caudatus } \\
*\end{array}$ & $\begin{array}{c}\text { A.hypochon- } \\
\text { driacus }^{\dagger}\end{array}$ & $\begin{array}{c}\text { A.caudatus } \\
\neq\end{array}$ & $\begin{array}{c}\text { Patrón } \\
\text { FAO }\end{array}$ \\
\hline TRP & N.D. & 3,6 & - & 1,0 \\
MET/CIS & $1,83^{\S}$ & 3,9 & 1,4 & 3,5 \\
TRE & 5,09 & 5,1 & 6,0 & 4,0 \\
ILE & 4,92 & 6,1 & 5,1 & 4,0 \\
VAL & 6,38 & 5,2 & 6,5 & 5,0 \\
LIS & 8,61 & 7,5 & 6,8 & 5,5 \\
FEN/TIR & 9,52 & 13,7 & 7,9 & 6,0 \\
LEU & 9,55 & 9,2 & 8,0 & 7,0 \\
\hline
\end{tabular}

N.D. No se determinó

* Proteína purificada de $35 \mathrm{kDa}$

+ Raina A (1992)

* Arnao I (1993)

$\S$ solo metionina 
to por Arnao y col. $\left({ }^{27}\right)$, a los que denominamos pico I y pico II de Sephadex G-75, representando este último aproximadamente $70 \%$ del total de la fracción.

La proteína de interés en el presente estudio fue eluida en el pico I, el cual sale con el Vo de la columna. Siendo el rango de fraccionamiento del Sephadex G-75 de 3000 a 80000 daltons, consideramos que la proteína, en su estado nativo, consta de varias subunidades, puesto que al realizar el análisis electroforético en condiciones denaturantes muestra un peso molecular de $35+/-1 \mathrm{kDa}$. Por otro lado, es conocido que las proteínas vegetales generalmente se encuentran asociadas formando estructuras multiméricas $\left({ }^{28,29}\right)$.

Las fracciones proteicas de los granos de amaranto muestran patrones electroforéticos característicos, con pequeñas diferencias de acuerdo al método de extracción y a las variedades usadas. El patrón electroforético de la fracción albúmina en estudio es semejante al obtenido por otros autores, quienes encuentran que en esta fracción hay dos grupos de polipéptidos: el grupo principal tiene pesos moleculares menores a $18 \mathrm{kDa}$ y el segundo, entre 25 y $42 \mathrm{kDa}\left({ }^{25}\right)$.

Shela Gorinstein $\left({ }^{26}\right)$, trabajando con cuatro especies de amaranto, informa que las principales subunidades de las albúminas, independiente de las especies, tienen pesos moleculares en el rango de $10,29,30$ y $37 \mathrm{kDa}$. Asimismo, Medrano y Ortega $\left.{ }^{(30}\right)$, haciendo el estudio de la fracción albúmina de cuatro ecotipos de $A$. caudatus procedentes de Huancayo, obtuvieron 19 bandas de polipéptidos, con pesos moleculares entre 80 y $10 \mathrm{kDa}$ y, una de las más abundantes, con peso molecular de 32 $\mathrm{kDa}$.

En el proceso de caracterización de las proteínas de amaranto, se ha utilizado diversas técnicas cromatográficas -filtración en gel, intercambio iónico, cromatografía de afinidad y ultracentrifugación-, a fin de establecer la naturaleza de sus constituyentes. Hasta la fecha, la única comunicación existente sobre purificación de proteínas de amaranto es el de Raina y Datta $\left({ }^{31}\right)$, quienes usando técnicas cromatográficas en DEAE-Sepharosa y gel filtración en Sephadex G-75 aislaron una proteína de
$35 \mathrm{kDa}$ de la fracción albúmina de Amaranthus hypochondriacus.

La gran mayoría de estudios llega hasta la caracterización de las fracciones y purificación parcial de algunas de ellas; tal es el caso de las glutelinas de $A$. hypochondriacus, de 67 y $50 \mathrm{kDa}\left({ }^{25}\right)$. También, cabe destacar que, usando una metodología similar a la utilizada en el presente trabajo, se ha aislado dos proteínas de 53,7 y 17,7 kDa, de la fracción globulina de Lupinus mutabilis $\left({ }^{28}\right)$ y una proteína de $22,5 \mathrm{kDa}$, de maca (Lepidium meyenni) $\left.{ }^{(32}\right)$.

Por muchos años, los fitomejoradores han tratado de elevar la calidad nutricional de las proteínas de semilla y actualmente con el auge de la biotecnología se ha renovado este interés. En general, los estudios realizados en cuanto a composición de aminoácidos de $A$. caudatus han sido llevados a cabo con el grano entero y en sus diferentes fracciones, existiendo algunas diferencias entre los investigadores respecto a qué fracción tiene el más alto contenido de lisina. La mayoría indica que es la fracción albúmina, cuyo nivel está alrededor de 6,7 moles \% ( $\left.{ }^{12,32}\right)$; para Segura Nieto y col. $\left({ }^{13}\right)$, son las globulinas con 7 moles $\%$.

En el presente estudio, el porcentaje de lisina expresada en moles $\%$, se va incrementando con el aislamiento de la proteína. Leucina y lisina, de la proteína de $35 \mathrm{kDa}$, tienen los más altos porcentajes por mol $(9,55 \%$ y $8,61 \%$, respectivamente) dentro de los aminoácidos esenciales y aspartato y glutamato $(12,12 \%$ y $10,07 \%$, respectivamente) dentro de los no esenciales. Existen pocos trabajos sobre la composición de aminoácidos de proteínas aisladas de la fracción albúmina. Uno de ellos es el de Raina y Datta $\left({ }^{31}\right)$, quienes aislaron una proteína de $35 \mathrm{kDa}$ de Amaranthus hipochondriacus, con 9,2 y 7,5 moles \% para leucina y lisina, respectivamente, observándose poca diferencia con los valores encontrados en el presente estudio. Asimismo, Arnao y col. ${ }^{34}$ ) comunican valores de 8,0 y 6,8 moles $\%$ para leucina y lisina, en una proteína aislada de esta misma fracción de Amaranthus caudatus.

Comparando con los datos informados por otros autores, encontramos que el nivel de lisina es mayor, 8,61 moles \% en nuestro caso y 7,5 y 6,8 moles \% según Raina y Arnao, respectivamente. 
En general, se podría decir que esta es una proteína con una composición en aminoácidos adecuada para la nutrición humana y muy semejante en su composición a lo comunicado por los autores antes mencionados.

Además, es importante observar que la leucina es uno de los aminoácidos limitantes en el grano entero $\left({ }^{5,6,22}\right)$. Sin embargo, en la proteína aislada, el porcentaje de este aminoácido esencial se incrementa. Otros aminoácidos esenciales presentes en la proteína de $35 \mathrm{kDa}$ purificada son treonina, isoleucina, valina, lisina, fenilalanina y tirosina, siendo esta composición superior a las recomendaciones establecidas por la FAO/OMS $\left({ }^{21}\right)$.

En general, las proteínas de almacenamiento de semilla tienen un alto valor nutricional y actualmente la proteína de la fracción albúmina de $A$. hypochondriacus (AmA1), así como la secuencia de ADN, han sido patentadas, esto es con el fin de producir plantas transgénicas con un alto valor nutricional, rica en aminoácidos esenciales.

Concluimos que la proteína de $35 \mathrm{kDa}$ aislada de la albúmina de Amaranthus caudatus es una proteína específica de la semilla, rica en lisina, con una composición en aminoácidos balanceada comparable a lo recomendado por la FAO/OMS y adecuada para la nutrición humana.

\section{AGRADECIMIENTOS}

Este trabajo fue parcialmente apoyado por CONCYTEC y el Concejo Superior de Investigación de la UNMSM.

\section{REFERENCIAS BIBLIOGRÁFICAS}

1. Ayala G, Vílchez W. Las repercusiones de los periodos agrícolas y los pisos agroecológicos del ande peruano sobre el consumo de alimentos y estado nutricional de la población. Lima, Perú: Empresa Científica Mundial; 1996.

2. National Research Council. Amaranth: Modern prospects for an ancient crop. Washington, DC: National Academy Press; 1984.

3. Teutonico R, Knoor D. Amaranth: Composition, properties, and applications of a rediscovered food crop. Food Technology. 1985;39(4):49-61.

4. Konishi Y, FumitaY, Ikeda K, Okuno K. Fuwa H. Isolation and characterization of globulin from seeds of Amaranthus hypochondriacus L. Agric Biol Chem. 1985;49:1453-9.

5. Duarte A, Jolk L, Carlsson R. Aminoacid composition of some Amaranthus sp grain proteins and of its fractions. Arch Latinoam Nutr. 1986;36:466-76.

6. Flores H. Teutonico R. III.5 Amaranths (Amaranthus spp): Potencial grain and vegetable crops. En: Bajai PS, Ed. Biotechnology in agriculture and forestery. Berlin: SpringerVerlag; 1986. p. 568-78.

7. Food and Nutrition Board. Recommended dietary allowances, $9^{\text {th }}$ ed. Washington, DC: National Academy of Sciences, National Research Council; 1980.

8. Deshpande P, Harper A, Quiros F, Elvehjem C. Further observations on the improvement of polished rice with protein and aminoacid supplements. J Nutr. 1966;57:415-28.

9. Howe E, Jansen G, Gilfillan E. Aminoacid supplementation of cereal grains as related to the world food supply. Am J Clin Nutr. 1965;16:315-20.

10. Rosenberg H, Culik R, Eckert R. Lysine and threonine supplementation of rice. Nutr. 1960;69:217-28.

11. Konishi Y, Yoshimoto N. Amaranth globulin as a heat stable emulsifying agent. Agric Biol Chem. 1989;53(12):33278.

12. Bressani R, Garcia L. Protein fractions in Amaranth grain and their chemical characterization. J Agric Food Chem. 1990;38:1205-9.

13. Segura-Nieto M, Vásquez-Sánchez N, Rubio-Velásquez $\mathrm{H}$, Olguín-Martínez L, Rodríguez-Nester C, Herrera-Estrella L. Characterization of Amaranth (Amaranthus hypochondriacus L.) seed proteins. J Agric Food Chem. 1992;40:1553-8.

14. Marcone M, Yada R. Study of the charge profile and covalent subunit association of the oligomeric seeds globulin from Amaranthus hypocondriacus. J Agric Food Chem. 1992;40:385-9.

15. Martínez N, Añon C. Composition and structural characterization of Amaranth proteins isolates. An electrophoretic and calorimetric study. J Agric Food Chem. 1996;44:2523-30.

16. Osborne T. The vegetable proteins. En: Monographs on Biochemistry. 2a ed. New York: Loongmans Green; 1924.

17. Harrington M. Elution of proteins from gels. In: Deutscher MP, editor. Methods in enzymology. New York: Academic Press; 1990. p. 182.

18. Laemmli UK. Cleavage of structural proteins during the assembly of the head of bacteriophage T4. Nature. 1970;227:680-5.

19. Bidlingmeyer BA, Cohen SA, Tarvin TL. Rapid analysis of aminoacids using pre-column derivatization. J Chromatogr. 1984;336:93-104.

20. Espinoza E. Caracterización bioquímica de las proteínas de Tropaeolum tuberosum. Tesis para optar el grado de Magíster. Universidad Nacional Mayor de San Marcos. Lima, Perú. 1998.

21. Food and Agriculture Organization of the United Nations. Amino acid content of foods and biological data on proteins. Rome: FAO, 1970 (Nutritional Studies No 24). 
22. Bressani R. El amaranto, una planta que ofrece grandes posibilidades de utilización agroindustrial. Alimentos. 1993;18(1):61.

23. Collazos y col. La composición de los alimentos peruanos. 5ta Ed. Lima, Perú: Ministerio de Salud; 1975.

24. Singhal R, Kulkarni P. Review: Amaranths, an underutilized resource. Int J Food Sci Technol. 1998;23:125-9.

25. Segura-Nieto M, Barba de la Rosa A, Paredes O. Biochemistry of Amaranth proteins. En: Paredes O. Amaranth, biology, chemistry and technology. Boca Ratón, Florida: CRC Press; 1994. p. 75-106.

26. Gorinstein S, Arnao I, Arruda P. Alcohol-soluble and total proteins from Amaranth seeds and their comparison with other cereals. J Agric Food Chem. 1991;39:848-50.

27. Arnao I. El amaranto (kiwicha): Un cultivo andino redescubierto hoy. Magistri et Doctores. 1993;3:8-10.

28. Barreto E. Estudio molecular de las proteínas de Lupinus mutabilis para su aplicación en biotecnología. Tesis para optar el título de Licenciada en Biología. Universidad Nacional Mayor de San Marcos, Lima, Perú. 1994.

29. Conger BV. Critical reviews in plant sciences. Boca Raton: CRC Press; 1996. p. 285-423.

30. Medrano M, Ortega B. Caracterización de cuatro ecotipos de Amaranthus caudatus "kiwicha" a través del análisis bromatológico, fraccionamiento proteico y electroforético. Tesis para optar el título de Químico Farmacéutico. Universidad Nacional Mayor de San Marcos. Lima, Perú. 1994.

31. Raina A, Datta A. Molecular cloning of a gene enconding a seed specific protein with nutricional and balanced aminoacid composition from Amaranthus. Proc Natl Acad Sci USA. 1992;80:11774-8

32. Monteghirfo M, Álvarez J, Espinoza S, Ramos Aliaga R. Caracterización bioquímica de las proteínas de Lepidium meyenni (maca) procedentes de Junín. En: I Jornadas de Investigación en Salud. Lima: Facultad de Medicina, UNMSM; 1999.

33. Gorinstein S, Moshe R, Greene L, Arruda P. Evaluation of four amaranthus species through protein electrophoretic patterns and their aminoacid composition. J Agric Food Chem. 1991;39:851-4.

34. Arnao I, Huerta D, Villanueva O. Purification of one protein with nutritionally balanced aminoacid composition from amaranthus. VIII Reunión de la Asociación Panamericana de Bioquímica y Biología Molecular. Pucón, Chile. 1996.

Manuscrito recibido el 17 de octubre de 2007 y aceptado para publicación el 13 de diciembre de 2007.

Correspondencia:

$\mathrm{Mg}$. Acela Inés Arnao Salas

Centro de Investigación de Bioquímica y Nutrición

Facultad de Medicina - UNMSM

Av. Grau 755. Lima 1, Perú

Correo-e: inesarnao@hotmail.com

orlyvv@yahoo.es 